\title{
SOME NEW ESTIMATIONS FOR THE HADAMARD PRODUCT OF A NONSINGULAR M-MATRIX AND ITS INVERSE
}

\author{
ZhengGe HuAng, Ligong WANG AND Zhong Xu
}

Abstract. In this paper, some new bounds for the minimum eigenvalue of the Hadamard product of a nonsingular $M$-matrix and its inverse are obtained, which improve some existing results. Finally, numerical examples are given to show that these bounds are better than some existing ones.

Mathematics subject classification (2010): 15A06, 15A18, 15A48.

Keywords and phrases: Nonsingular $M$-matrix, Hadamard product, inequality, minimum eigenvalue.

\section{REFERENCES}

[1] A. Berman And R. J. Plemmons, Nonnegative Matrices in the Mathematical Sciences, SIAM, Philadelphia, 1979.

[2] R. A. Horn And C. R. Johnson, Topics in Matrix Analysis, Cambridge Press, New York, 1991.

[3] M. Fiedler AND T. L. MARKHAM, An inequality for the Hadamard product of an M-matrix and its inverse, Linear Algebra Appl. 101, 1 (1988), 1-8.

[4] J. ZHANG, J. Z. LIU AND G. TU, The improved disc theorems for the Schur complements of diagonally dominant matrices, J. Inequal. Appl. 2, 1 (2013), 1-16.

[5] M. Fiedler, T. L. Markham And M. Neumann, A trace inequality for M-matrix and the symmetrizability of a real matrix by a positive diagonal matrix, Linear Algebra Appl. 71, 1 (1985), 81-94.

[6] S. C. CHEN, A lower bound for the minimum eigenvalue of the Hadamard product of matrices, Linear Algebra Appl. 378, 1 (2004), 159-166.

[7] Y. Z. Song, On an inequality for the Hadamard product of an M-matrix and its inverse, Linear Algebra Appl. 305, 1 (2000), 99-105.

[8] X. R. Yong, Proof of a conjecture of Fiedler and Markham, Linear Algebra Appl. 320, 1 (2000), $167-171$.

[9] H. B. Li, T. Z. HuAng, S. Q. Shen AND H. Li, Lower bounds for the minimum eigenvalue of Hadamard product of an M-matrix and its inverse, Linear Algebra Appl. 420, 1 (2007), $235-247$.

[10] Y. T. Li, F. B. Chen AND D. F. WANG, New lower bounds on eigenvalue of the Hadamard product of an M-matrix and its inverse, Linear Algebra Appl. 430, 4 (2009), 1423-1431.

[11] G. H. Cheng, Q. TAN AND Z. D. WANG, Some inequalities for the minimum eigenvalue of the Hadamard product of an M-matrix and its inverse, J. Inequal. Appl. 65, 1 (2013), 1-9.

[12] D. M. Zhou, G. L. Chen, G. X. Wu And X. Y. Zhang, Some inequalities for the Hadamard product of an M-matrix and an inverse M-matrix, J. Inequal. Appl. 16, 1 (2013), 1-10.

[13] Y. T. Li, F. WAng, C. Q. Li And J. X. ZhaO, Some new bounds for the minimum eigenvalue of the Hadamard product of an M-matrix and an inverse M-matrix, J. Inequal. Appl. 480, 1 (2013), 1-8.

[14] F. B. CHEN, New inequalities for the Hadamard product of an M-matrix and its inverse, J. Inequal. Appl. 35, 1 (2015), 1-12.

[15] Z. XU, Q. LU , K. Y. Zhang And X. H. An, Theory and Applications of H-matrices, Science Press, Beijing, 2013.

[16] X. Y. Yong, On a conjecture of Fiedler and Makham, Linea Algebra Appl. 288, 1 (1999), 259-267.

[17] R. A. Horn And C. R. Johnson, Matrix Analysis, Cambridge University Press, Cambridge, 1985. 